\title{
Psychosocial Stress and Salivary Cortisol in Older People: A Brief Review
}

Julian CL Lai*

Department of Applied Social Studies, City University of Hong Kong, Hong Kong, China

*Corresponding author: Julian CL Lai, Department of Applied Social Studies, City University of Hong Kong, Hong Kong, China, Tel: +85234424306 ; Fax: +852 34420283; E-mail: ssjulwin@cityu.edu.hk, ssjulwin@netvigator.com

Rec date: Mar 28, 2014, Acc date: Apr 24, 2014, Pub date: Apr 29, 2014

Copyright: @ 2014 Lai JCL, et al. This is an open-access article distributed under the terms of the Creative Commons Attribution License, which permits unrestricted use, distribution, and reproduction in any medium, provided the original author and source are credited.

\begin{abstract}
The Hypothalamic-Pituitary-Adrenal (HPA) axis is crucial for homeostatic and allostatic adjustments to internal and external challenges. However, how aging affects this neuroendocrine axis is still incompletely understood. Being the end-product of the HPA axis, cortisol has been extensively studied for the last two decades because an increased cortisol response to psychosocial challenge has been hypothesized to be a risk factor for developing a number of age-related disorders in humans. This hypothesis has been addressed by separate lines of research focusing on the impact of acute laboratory stressors and chronic natural stressors on cortisol secretion in the elderly. This paper summarizes major findings generated by the aforementioned lines of research with a focus on studies examining salivary cortisol. It is concluded that although age does not have a consistent effect on cortisol response to acute laboratory stressors, evidence supporting an age effect on basal cortisol secretion is emerging. Moreover, the age-cortisol relationship is modulated by chronic stress and other psychosocial factors. Further research is warranted to throw more light on these preliminary findings.
\end{abstract}

Keywords: Salivary cortisol; Older people; Cortisol awakening response (CAR); Trier Social Stress Test (TSST); Hypothalamicpituitary-adrenal (HPA) axis; Stress

\section{Introduction}

Aging has been assumed to be associated with a decline in the capacity to adapt to stressful forces in life, which is marked by an increase in cortisol response to stressful challenges [1-3]. This assumption has been one of the main driving forces of research on psychosocial stress and cortisol secretion in aging populations. Being the end-product of the HPA axis, cortisol has been studied extensively because loss of diurnal regulation has been seen as crucial to the aging process and the onset of frailty [4-6]. Recent evidence suggests that increase in cortisol secretion is a key factor determining immuosenescence [7] and a lower level of cortisol is associated with longevity [8].

However, findings generated in the last two decades fail to provide unequivocal support to the aforementioned assumption. Mixed findings may partly reflect the remarkably heterogeneous responses of the aged population to stressful challenges, which remain to be delineated more clearly. It is possible that some old people may age more successfully than others such that they are able to remain biologically younger than their peers $[9,10]$. Keeping the aforementioned issues in mind, I review recent findings on the relationship between psychosocial stress and cortisol regulation in aging so as to arrive at a better understanding of the multitude of factors that determine reactivity of the HPS axis in the aged populations. Although there are already a handful of recent reviews on psychosocial stress and cortisol [11,12], none of these focuses specifically on the elderly and age effect. This review is expected to fill this gap in the literature. The focus of this paper will be on studies examining salivary cortisol for two reasons. First, as cortisol in saliva does not bind to corticosteroid binding globulins as it does in the blood, salivary cortisol can be considered as a more reliable indicator of free or active cortisol than serum cortisol [13]. Second, the use of salivary cortisol as a biomarker of stress has become increasingly common in geriatric research. Therefore, focusing on studies examining salivary cortisol is more likely to lead to conclusions that reflect the current knowledge accurately.

\section{Control of cortisol secretion}

Cortisol has profound effects on glucose metabolism, helps to make fat available for energy, and modulates the immune responses, among other physiological functions that it serves [14]. The role cortisol plays in physiologic function is so crucial that as pointed out by Clow, Thorn, Evans, and Hucklebridge (2004) [15], we cannot survive without this hormone. The age-related increase in cortisol secretion has also been hypothesized to be a key determinant of the decline in immune function associated with aging [7].

The release of cortisol into the bloodstream is a result of a cascade initiated in the hypothalamus. Specifically, the corticotropin-releasinghormone $(\mathrm{CRH})$ is synthesized in and released by the paraventricular nucleus (PVN) of the hypothalamus into the portal blood circulation. When the $\mathrm{CRH}$ reaches the anterior pituitary gland, it stimulates the secretion of adrenocorticotropic hormone (ACTH). ACTH enters the systemic blood circulation, and stimulates the adrenal cortex to synthesize and secret glucocorticoids, with cortisol being the main glucocorticoid in humans [16]. Exposure to psychosocial stress intensifies the activity of the HPA axis, which results in an increase in the secretion of cortisol to maintain homeostasis. This increase in cortisol secretion is controlled by a negative feedback mechanism which detects increased cortisol level by receptors located in multiple brain regions such as the hippocampus, the hypothalamus, and the pituitary gland $[17,18]$. As successful adaptation involves both the mounting of a cortisol response to stressors and an appropriate control of this response, deficiencies in either one of the two mechanisms may have pathogenic consequences. 


\section{Measuring cortisol secretory activity}

One of the major challenges of studying the effect of psychosocial stress on cortisol secretion is the establishment of reliable indices of cortisol secretory activity. The basal level of HPA axis activity follows a well-documented diurnal rhythm with several episodes of highamplitude secretion. Cortisol secretion peaks within 60 minutes after waking and declines thereafter until reaching the nadir around midnight [17]. Recent evidence suggests that the cortisol awakening response (CAR), which is marked by an increase from $50 \%$ to $150 \%$ within the first 30 minutes after waking up in the morning [19], is a reliable marker of HPA axis activity [15]. This distinct component of the cortisol diurnal rhythm has been shown to have a salient genetic component in twin studies $[20,21]$, and is sensitive to chronic stress in the elderly [22,23]. Recent evidence suggests that in addition to being a measure of the activity of the HPA axis, the CAR also involves in the mobilization of energy for the transition from sleep to wakefulness, which is partly mediated by the suprachiasmatic nucleus [24].

The CAR can be divided into two separate components: the area under the curve with respect to ground $\left(\mathrm{AUC}_{\mathrm{G}}\right)$ and the area under the curve with respect to increase $\left(\mathrm{AUC}_{\mathrm{I}}\right)$ [25]. The former captures the overall concentration whereas the latter indexes the increase from after post-awakening. However, a number of researchers have argued that CAR should restrictively refer to the increase in cortisol from awakening and be operationalized by the $\mathrm{AUC}_{\mathrm{I}}$ or similar indices [14].

Other reliable markers of the activity of the HPA axis that have been adopted in geriatric research are (1) the total diurnal secretion or cortisol exposure throughout the day, and (2) the diurnal decline. The former refers to the overall concentration of cortisol over the course of a day, and has been operationalized as area under the concentrationtime curve (AUC) [26]. The second marker, diurnal decline has been operationalized in prior research as a decrease in the cortisol levels measured at different times during the day [27]. For example, the diurnal slope which is computed by dividing the difference between the cortisol level at 30 minutes and 12 hours post-awakening by the time interval between these two samples [28].

\section{Cortisol response to laboratory stressors}

\section{The Trier Social Stress Test}

A number of studies have examined the difference in cortisol response to laboratory stressors between younger and older participants [11,12]. Being the most commonly used laboratory stressor, the Trier Social Stress Test (TSST) consists of a preparation period, a public speaking and a mental arithmetic task in front of an audience [29]. Lasting for about 15 minutes, the TSST has been shown to reliably elicit the strongest cortisol response in comparison to other protocols $[30,31]$ a two- to three-fold rise in cortisol is observed in $70 \%$ to $85 \%$ of participants exposed to the stressor. The cortisol response to the TSST exhibits a prototypical pattern of change over time: increases steadily from the onset of stressor, peaks at about 20 minutes, and returns to pre-stress level within 45 minutes [11]. This is most commonly operationalized as area under the concentration-time curve (Figure 1), which implies that saliva or blood samples must be collected at multiple time points to fully capture the entire stress response. The cortisol response to TSST is determined by genetic factors as shown in twin studies [31] as well as gene studies on polymorphisms in HPA axis related genes [32].

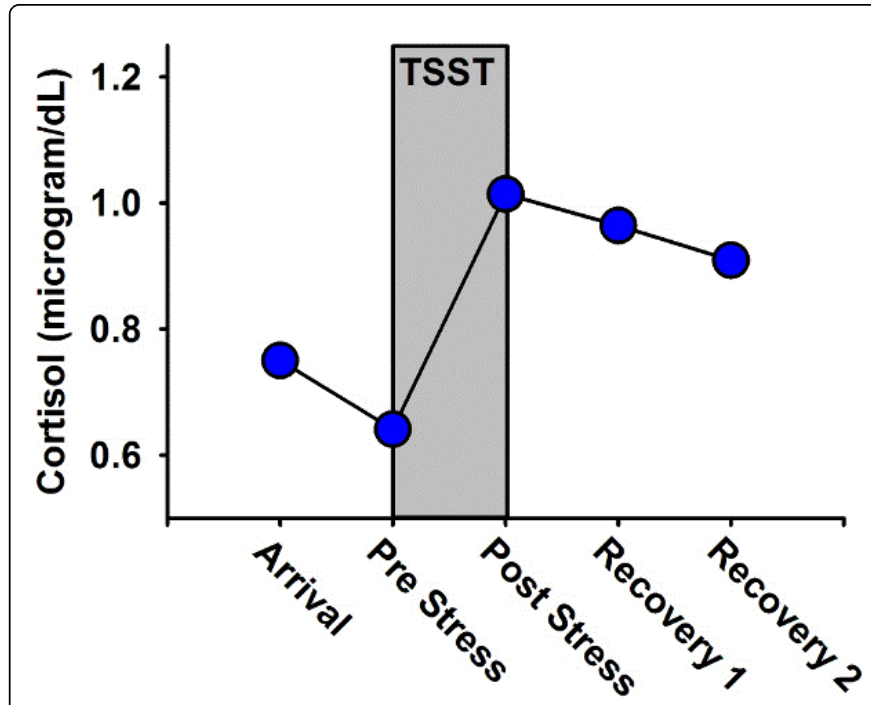

Figure 1: Hypothetical Cortisol Data in Response to the TSST

Note: TSST $=$ Trier Social Stress Test. Adapted from Birkett (2011) [57]. The Trier Social Stress Test Protocol for inducing psychological stress. Journal of Visualized Experiments, 56, e3238. (doi: $10.3791 / 3238)$

\section{Age difference in cortisol response to the TSST}

On the basis of findings reported in prior studies, a significantly greater increase in cortisol response was not observed consistently in the older group. In particular, in studies using a younger group whose mean age was below 30 yrs [33,34], the hypothesized age effect was not demonstrated, which is consistent with data reported by two earlier studies $[35,36]$. However, when the older group was compared to an older younger group (mean age $=50 \mathrm{yrs}$ ) [37], an accentuated cortisol response was observed in the younger instead of the older group. Apparently, the conclusions that can be drawn from these crosssectional findings are very limited, given the small effect sizes of the age effect (the absolute sizes of $d$ ranged from .29 to .42) [2].

The interpretability of the aforementioned findings is further curtailed by the absence of a proper control condition in prior studies. A proper control group is one that requires participants to engage in the public speaking and mental math task without being evaluated by a panel of judges. Any cortisol responses to this condition could only be attributed to the cognitive demand of the tasks. This point is important because cognitive tasks without explicit social-evaluative threat have been found to induce higher cortisol response in older participants [38]. In other words, without a control group, researchers cannot attribute observed age differences in cortisol response specifically to the uncontrollable social-evaluative threat that constitutes the major source of stress of the TSST [39].

\section{Factors modifying the cortisol response to TSST}

As pointed out in recent reviews, age is only one of the numerous factors that determine the cortisol response to TSST $[11,12,40]$. Genetic and psychological factors could play a more important role in explaining the variation of cortisol response to TSST in older people. Findings from a recent study with 97 healthy older participants (mean age $=61.15$ years) point clearly to the significant influence of 
polymorphism in the HTR1A gene encoding serotonin receptor 1A (5HT1A) on cortisol response to TSST: the G allele is associated with an attenuated response [41]. It has also been shown in the same study that stressful events experienced during childhood and adolescence are associated negatively with the cortisol response to the TSST. These recent findings suggest that the cortisol response to the TSST in the elderly cannot be accurately assessed without taking into account the effects of the aforementioned factors.

\section{Chronic stress and diurnal cortisol rhythms in aging}

The conjecture that aging is associated with elevated diurnal cortisol seems to be supported by available evidence. A number of cross-sectional studies have shown a positive association between age and diurnal secretion of cortisol [42-44]. Following a group of 51 healthy older participants over 6 years, Lupien et al. (1996) [45] observed an increase in 24-hour plasma cortisol concentrations over 6 years in participants. In line with this, in a recent longitudinal study with a larger sample of older participants $(\mathrm{N}=157)$, diurnal cortisol concentrations were found to increase significantly over a period of 4 years [46]. Impairment in the negative feedback of the HPA axis in old age may explain this observation because an elevation of CRH has been observed in older people in response to the dexamethasone suppression test $[47,48]$. Taken together, these findings can be taken to imply that aging is associated with an increase in tissue exposure to cortisol.

With respect to aging and CAR, although it has been reported that there is a trend for age to be associated with a lower CAR in older participants [49], age has also been found to be associated with a greater CAR [50-52], or have no significant relationship with CAR $[9,53]$. The reasons for mixed findings are not immediately apparent but can be attributed to differences in operationalization of CAR, ages of participants, influences of state-dependent variables, and compliance with the study protocol. Compliance becomes particularly important for accurate assessment of the CAR because delays greater than 5 minutes between awakening and collection of the first saliva sample can lead to inaccurate estimation [18]. The compliance issue becomes most problematic when awakening and saliva sample collection times are monitored by self-reports instead of objectively with electronic devices as being done in most studies.

A limited amount of data shows that chronic stressors such as bereavement or caregiving in older people lead to a blunted cortisol concentrations [23]. Older people with anxiety disorders have been found to exhibit a blunted CAR in comparison to their healthy peers [22]. In line with this, Holocaust survivors with PTSD also exhibit a blunted morning cortisol response compared to those survivors without PTSD [54].

The more critical issue of whether age-related changes in the diurnal cortisol secretion are modified by chronic stress and other factors has been addressed in a handful of recent studies. In particular, the positive association between age and nocturnal cortisol levels has been shown to become stronger with increasing anxiety levels in participants [43]. Moreover, low self-esteem accentuates the negative association between age and CAR [49] whereas longer sleep duration in older people tends to counteract the elevated diurnal secretion in aging [46]. These findings imply that the age effect on basal cortisol is moderated by psychosocial factors. Further research to throw more light on this pattern of relationships is warranted.

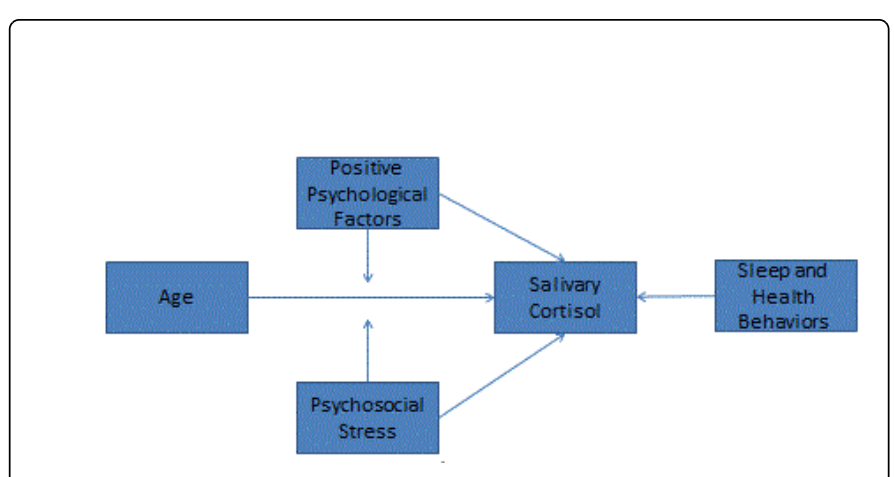

Figure 2: A Conceptual Framework Relating Age and Psychosocial Factors to Salivary Cortisol in Older People

Note: The hypothesized longitudinal effect of age on salivary cortisol is exacerbated by psychosocial stress and mitigated by positive psychological factors. Aging is expected to have negative neuroendocrine effects when older individuals are exposed to high level of stress without sufficient protection conferred by positive psychological factors. Sleep and other health behaviors are treated as confounding variables in the model.

\section{Conclusions}

Findings reviewed earlier suggest that age does not significantly affect cortisol response to acute laboratory stressors. Although aging is associated with complex changes in the HPA axis [1], these changes do not seem to manifest in the cortisol response to acute psychosocial stress in laboratory settings. In other words, alterations in the cortisol response to acute psychosocial stress is not necessary a consequence of aging. Further pursuit along this line of research does not seem to be promising.

On the other hand, available evidence tends to suggest a positive association between age and diurnal cortisol secretion and moderating effects of stress and other factors on this relationship. However, as the amount of supporting data is still limited, further research is warranted to arrive at more concrete conclusions. Future research will be benefited by adopting a conceptual framework that is informed by some of the most recent findings. As illustrated in Figure 2, the focus of the framework is on the longitudinal impact of age on different indices of the diurnal cortisol profile in the elderly. Special attention is given to the potential moderating effect of psychosocial factors. Moderators are not restricted to stress but include factors that confer resilience to stressful encounters because data showing the beneficial neuroendocrine effects of the resilience factors in the elderly are mounting $[9,10,53,55]$. As sleep and health behaviors exert detectable effects on cortisol secretion [46,56], these confounding variables must be properly controlled for accurate assessment of the effects of age and psychosocial stress.

\section{References}

1. Chahal HS, Drake WM (2007) The endocrine system and ageing. J Pathol 211: 173-180.

2. Otte C, Hart S, Neylan TC, Marmar CR, Yaffe K, et al. (2005) A metaanalysis of cortisol response to challenge in human aging: importance of gender. Psychoneuroendocrinology 30: 80-91. 
3. McEwen BS (2002) Sex, stress and the hippocampus: allostasis, allostatic load and the aging process. Neurobiol Aging 23: 921-939.

4. Fried LP, Walston J (1999) Frailty and failure to thrive. In: Hazzard, WR Blass, JP Ettinger, W Halter, JB Ouslander, JG (Eds.), Principals of Geriatric Medicine and Gerontology. McGraw-Hill: New York 1387-1402.

5. Sapolsky RM, Krey LC, McEwen BS (1986) The neuroendocrinology of stress and aging: the glucocorticoid cascade hypothesis. Endocr Rev 7: 284-301.

6. Spiegel D, Sephton SE (2001) Psychoneuroimmune and endocrine pathways in cancer: effects of stress and support. Semin Clin Neuropsychiatry 6: 252-265.

7. Buford TW, Willoughby DS (2008) Impact of DHEA(S) and cortisol on immune function in aging: a brief review. Appl Physiol Nutr Metab 33: 429-433.

8. Noordam R, Jansen SW, Akintola AA, Oei NY, Maier AB, et al. (2012) Familial longevity is marked by lower diurnal salivary cortisol levels: the Leiden Longevity Study. PLoS One 7: e31166.

9. Lai JCL, Chong AML, Siu OT, Evans P, Chan CLW, et al. (2010) Humor attenuates the cortisol awakening response in healthy older men Biological Psychology 84: 375-380.

10. Lai JC, Chong AM, Siu OT, Evans P, Chan CL, et al. (2012) Social network characteristics and salivary cortisol in healthy older people. Scientific World Journal 2012: 929067.

11. Foley P, Kirschbaum C (2010) Human hypothalamus-pituitary-adrenal axis responses to acute psychosocial stress in laboratory settings. Neurosci Biobehav Rev 35: 91-96.

12. Kudielka BM, Wüst S (2010) Human models in acute and chronic stress: assessing determinants of individual hypothalamus-pituitary-adrenal axis activity and reactivity. Stress 13: 1-14.

13. Kumsta R, Entringer S, Hellhammer DH, Wüst S (2007) Cortisol and ACTH responses to psychosocial stress are modulated by corticosteroid binding globulin levels. Psychoneuroendocrinology 32: 1153-1157.

14. Smyth N, Hucklebridge F, Thorn L, Evans P, Clow A (2013) Salivary cortisol as a biomarker in social science research. Social and Personality Psychology Compass 7: 605-625.

15. Clow A, Thorn L, Evans P, Hucklebridge F (2004) The awakening cortisol response: methodological issues and significance. Stress 7: 29-37.

16. Miller DB, O'Callaghan JP (2002) Neuroendocrine aspects of the response to stress. Metabolism 51: 5-10.

17. Fries E, Dettenborn L, Kirschbaum C (2009) The cortisol awakening response (CAR): facts and future directions. Int J Psychophysiol 72: 67-73.

18. Smyth N, Clow A, Thorn L, Hucklebridge F, Evans P (2013) Delays of 5-15 min between awakening and the start of saliva sampling matter in assessment of the cortisol awakening response. Psychoneuroendocrinology 38: 1476-1483.

19. Pruessner JC, Wolf OT, Hellhammer DH, Buske-Kirschbaum A, von Auer K, et al. (1997) Free cortisol levels after awakening: a reliable biological marker for the assessment of adrenocortical activity. Life Sci 61: 2539-2549.

20. Kupper N, de Geus EJ, van den Berg M, Kirschbaum C, Boomsma DI, et al. (2005) Familial influences on basal salivary cortisol in an adult population. Psychoneuroendocrinology 30: 857-868.

21. Wüst S, Federenko I, Hellhammer DH, Kirschbaum C (2000) Genetic factors, perceived chronic stress, and the free cortisol response to awakening. Psychoneuroendocrinology 25: 707-720.

22. Hek K, Direk N, Newson RS, Hofman A, Hoogendijk WJ, et al. (2013) Anxiety disorders and salivary cortisol levels in older adults: a population-based study. Psychoneuroendocrinology 38: 300-305.

23. Okun M, Reynolds C, Monk T, Hall M (2008) Variability in cortisol among the aging: Relationships to chronic stress and good stress. Brain, Behavior, and Immunity 22: 8-9.
24. Clow A, Hucklebridge F, Stalder T, Evans P, Thorn L (2010) The cortisol awakening response: more than a measure of HPA axis function. Neurosci Biobehav Rev 35: 97-103.

25. Pruessner JC, Kirschbaum C, Meinlschmidt G, Hellhammer D (2003) Two formulas for computation of the area under the curve represent measures of total hormone concentration versus time-dependent change. Psychoneuroendocrinology 28: 916-931.

26. Direk N, Newson RS, Hofman A, Kirschbaum C, Tiemeier H (2011) Short and long-term effects of smoking on cortisol in older adults. Int J Psychophysiol 80: 157-160.

27. Edwards S, Hucklebridge F, Clow A, Evans P (2003) Components of the diurnal cortisol cycle in relation to upper respiratory symptoms and perceived stress. Psychosomatic Medicine 65: 320-327.

28. Adam EK, Kumari M (2009) Assessing salivary cortisol in large-scale, epidemiological research. Psychoneuroendocrinology 34: 1423-1436.

29. Kirschbaum C, Pirke KM, Hellhammer DH (1993) The 'Trier Social Stress Test'-A tool for investigating psychobiological stress responses in a laboratory setting. Neuropsychobiology 28: 76-81.

30. Dickerson SS, Kemeny ME (2004) Acute stressors and cortisol responses: a theoretical integration and synthesis of laboratory research. Psychol Bull 130: 355-391.

31. Federenko IS, Nagamine M, Hellhammer DH, Wadhwa PD, Wüst $S$ (2004) The heritability of hypothalamus pituitary adrenal axis responses to psychosocial stress is context dependent. J Clin Endocrinol Metab 89: 6244-6250.

32. Wüst S, Van Rossum EF, Federenko IS, Koper JW, Kumsta R, et al. (2004) Common polymorphisms in the glucocorticoid receptor gene are associated with adrenocortical responses to psychosocial stress. J Clin Endocrinol Metab 89: 565-573.

33. Kudielka BM, Buske-Kirschbaum A, Hellhammer DH, Kirschbaum C (2004) HPA axis responses to laboratory psychosocial stress in healthy elderly adults, younger adults, and children: impact of age and gender. Psychoneuroendocrinology 29: 83-98.

34. Rohleder N, Kudielka BM, Hellhammer DH, Wolf JM, Kirschbaum C (2002) Age and sex steroid-related changes in glucocorticoid sensitivity of pro-inflammatory cytokine production after psychosocial stress. J Neuroimmunol 126: 69-77.

35. Kudielka BM, Schmidt-Reinwald AK, Hellhammer DH, Kirschbaum C (1999) Psychological and endocrine responses to psychosocial stress and dexamethasone/corticotropin-releasing hormone in healthy postmenopausal women and young controls: the impact of age and a two-week estradiol treatment. Neuroendocrinology 70: 422-430.

36. Kudielka BM, Schmidt-Reinwald AK, Hellhammer DH, Schürmeyer T, Kirschbaum C (2000) Psychosocial stress and HPA functioning: no evidence for a reduced resilience in healthy elderly men. Stress 3: 229-240.

37. Nicolson N, Storms C, Ponds R, Sulon J (1997) Salivary cortisol levels and stress reactivity in human aging. J Gerontol A Biol Sci Med Sci 52: M68-75.

38. Gotthardt U, Schweiger U, Fahrenberg J, Lauer CJ, Holsboer F, et al. (1995) Cortisol, ACTH, and cardiovascular response to a cognitive challenge paradigm in aging and depression. Am J Physiol 268: R865-873.

39. Het S, Rohleder N, Schoofs D, Kirschbaum C, Wolf OT (2009) Neuroendocrine and psychometric evaluation of a placebo version of the 'Trier Social Stress Test'. Psychoneuroendocrinology 34: 1075-1086.

40. Allen AP, Kennedy PJ, Cryan JF, Dinan TG, Clarke G (2014) Biological and psychological markers of stress in humans: focus on the Trier Social Stress Test. Neurosci Biobehav Rev 38: 94-124.

41. Armbruster D, Mueller A, Strobel A, Lesch KP, Brocke B, et al. (2011) Predicting cortisol stress responses in older individuals: influence of serotonin receptor 1A gene (HTR1A) and stressful life events. Horm Behav 60: 105-111.

42. Ice GH (2005) Factors influencing cortisol level and slope among community dwelling older adults in Minnesota. J Cross Cult Gerontol 20: 91-108. 
Citation: Lai JCL (2014) Psychosocial Stress and Salivary Cortisol in Older People: A Brief Review. Aging Sci 2: 120. doi: 10.4172/2329-8847.1000120

Page 5 of 5

43. Hartaigh B, Loerbroks A, Thomas GN, Engeland CG, Hollands MA, et al. (2012) Age-dependent and -independent associations between depression, anxiety, DHEAS, and cortisol: from the MIPH Industrial Cohort Studies (MICS). Psychoneuroendocrinology 37: 929-936.

44. Nater UM, Hoppmann CA, Scott SB (2013) Diurnal profiles of salivary cortisol and alpha-amylase change across the adult lifespan: Evidence from repeated daily life assessments. Psychoneuroendocrinology 38: 3167-3171.

45. Lupien S, Lecours AR, Schwartz G, Sharma S, Hauger RL, et al. (1996) Longitudinal study of basal cortisol levels in healthy elderly subjects: evidence for subgroups. Neurobiol Aging 17: 95-105.

46. Rueggeberg R, Wrosch C, Miller GE (2012) Sleep duration buffers diurnal cortisol increases in older adulthood. Psychoneuroendocrinology 37: 1029-1038.

47. Heuser IJ, Gotthardt U, Schweiger U, Schmider J, Lammers CH, et al (1994) Age-associated changes of pituitary-adrenocortical hormone regulation in humans: importance of gender. Neurobiol Aging 15: 227-231.

48. Parker CR Jr, Slayden SM, Azziz R, Crabbe SL, Hines GA, et al. (2000) Effects of aging on adrenal function in the human: responsiveness and sensitivity of adrenal androgens and cortisol to adrenocorticotropin in premenopausal and postmenopausal women. J Clin Endocrinol Metab 85: 48-54.

49. Pruessner JC, Baldwin MW, Dedovic K, Renwick R, Mahani NK, et al. (2005) Self-esteem, locus of control, hippocampal volume, and cortisol regulation in young and old adulthood. Neuroimage 28: 815-826.
50. Almeida DM, Piazza JR, Stawski RS (2009) Interindividual differences and intraindividual variability in the cortisol awakening response: an examination of age and gender. Psychol Aging 24: 819-827.

51. Dmitrieva NO, Almeida DM, Dmitrieva J, Loken E, Pieper CF (2013) A day-centered approach to modeling cortisol: diurnal cortisol profiles and their associations among U.S. adults. Psychoneuroendocrinology 38: 2354-2365.

52. Kumari M, Badrick E, Sacker A, Kirschbaum C, Marmot M, et al. (2010) Identifying patterns in cortisol secretion in an older population. Findings from the Whitehall II study. Psychoneuroendocrinology 35: 1091-1099.

53. O'Donnell K, Badrick E, Kumari M, Steptoe A (2008) Psychological coping styles and cortisol over the day in healthy older adults. Psychoneuroendocrinology 33: 601-611.

54. Yehuda R, Golier JA, Harvey PD, Stavitsky K, Kaufman S, et al. (2005) Relationship between cortisol and age-related memory impairments in Holocaust survivors with PTSD. Psychoneuroendocrinology 30: 678-687.

55. Lai JC, Evans PD, Ng SH, Chong AM, Siu OT, et al. (2005) Optimism, positive affectivity, and salivary cortisol. Br J Health Psychol 10: 467-484.

56. Fukuda S, Morimoto K (2001) Lifestyle, stress and cortisol response: Review II: Lifestyle. Environ Health Prev Med 6: 15-21.

57. Birkett MA (2011) The Trier Social Stress Test protocol for inducing psychological stress. J Vis Exp . 